\title{
Herpes simplex virus type 1 UL14 tegument protein regulates intracellular
} compartmentalization of major tegument protein
VP16

Akane Ohta ${ }^{1}$, Yohei Yamauchi ${ }^{2}$, Yoshifumi Muto ${ }^{1}$, Hiroshi Kimura ${ }^{1}$ and Yukihiro Nishiyama ${ }^{1 *}$

\begin{abstract}
Background: Herpes simplex virus type 1 (HSV-1) has a complicated life-cycle, and its genome encodes many components that can modify the cellular environment to facilitate efficient viral replication. The protein UL14 is likely involved in viral maturation and egress (Cunningham C. et al), and it facilitates the nuclear translocation of viral capsids and the tegument protein VP16 during the immediate-early phase of infection (Yamauchi Y. et al, 2008). UL14 of herpes simplex virus type 2 exhibits multiple functions (Yamauchi Y. et al, 2001, 2002, 2003).
\end{abstract}

Methods: To better understand the function(S) of UL14, we generated VP16-GFP-incorporated UL14-mutant viruses with either single (K51M) or triple (R60A, R64A, E68D) amino acid substitutions in the heat shock protein (HSP)-like sequence of UL14. We observed the morphology of cells infected with UL14-null virus and amino acid-substituted UL14-mutant viruses at different time points after infection.

Results: UL14(3P)-VP16GFP and UL14D-VP16GFP (UL14-null) viruses caused similar defects with respect to growth kinetics, compartmentalization of tegument proteins, and cellular morphology in the late phase. Both the UL14DVP16GFP and UL14(3P)-VP16GFP viruses led to the formation of an aggresome that incorporated some tegument proteins but did not include nuclear-egressed viral capsids.

Conclusions: Our findings suggest that a cluster of charged residues within the HSP-like sequence of UL14 is important for the molecular chaperone-like functions of UL14, and this activity is required for the acquisition of functionality of VP16 and UL46. In addition, UL14 likely contributes to maintaining cellular homeostasis following infection, including cytoskeletal organization. However, direct interactions between UL14 and VP16, UL46, or other cellular or viral proteins remain unclear.

Keywords: Herpes simplex virus, UL14, VP16, molecular chaperone, heat shock proteins, aggresome

\section{Background}

Herpes simplex virus (HSV) type $1(\mathrm{HSV}-1)$ is a large, enveloped DNA virus whose genome contains at least 74 genes [1,2]. Previous studies showed that approximately half of these genes are unessential for virus replication in cell culture [2]. However, the dispensable gene products may be important for viral growth and propagation in vivo [3]. Additionally, 40 core genes have been

\footnotetext{
* Correspondence: ynishiya@med.nagoya-u.ac.jp

1 Department of Virology, Graduate School of Medicine, Nagoya University,

Tsurumai-cho 65, Showa-ku, Nagoya 466-8550, Japan

Full list of author information is available at the end of the article
}

identified based on significant genomic homology among the three Herpesviridae subfamilies[4]. However, one-third of the core genes are dispensable[3], and their functions remain unknown. To gain a comprehensive understanding of the HSV lifecycle, it is important to investigate the functions of these accessory genes both in vitro and in vivo.

UL14 is an established core gene but it is dispensable for viral replication in vitro. UL14 is a $32-\mathrm{kDa}$ protein expressed in the late phase of viral infection, after viral DNA synthesis has occurred [5,6]. The coding region of HSV-1 UL14 overlaps that of UL13, but most conserved

\section{Biomed Central}

(c) 2011 Ohta et al; licensee BioMed Central Ltd. This is an Open Access article distributed under the terms of the Creative Commons Attribution License (http://creativecommons.org/licenses/by/2.0), which permits unrestricted use, distribution, and reproduction in any medium, provided the original work is properly cited. 
residues are located in the nonoverlapping region. The overlapping region encodes a variable-length $\mathrm{C}$-terminal domain that is poorly conserved [6]. We previously showed that the UL14 protein of HSV type 2 (HSV-2) facilitates the translocation of both capsid protein VP26 (which is encoded by the UL35 gene) and UL33 protein into the nucleus in cells co-expressing these proteins in the absence of viral infection[7]. UL14 also exhibits heat shock protein (HSP)-like functions when expressed alone in cells. Additionally, a region that is highly conserved between $\mathrm{HSV}-1$ and HSV-2, especially ${ }_{60}$ RLKSRARLE $_{68}$, is similar to an $\alpha$ helix contained within the substrate-binding region of Hsp70 (Figure 1A) [8,9]. UL14 undergoes intercellular trafficking and prevents apoptosis induced by chemical and hypertonic stress $[10,11]$. Finally, upon infection, UL14 is incorporated into the virion as a minor tegument protein, and it is required for efficient growth of the virus $[5,6]$.
VP16 is an abundant $65-\mathrm{kDa}$ phosphoprotein that is synthesized during the late phase of infection, and it is subsequently packaged into virions as a major tegument protein [12-15]. VP16 acts during the earliest stages of infection in concert with HCF-1 and Oct-1 to induce transcription of the viral immediate-early (IE) genes, thereby facilitating the onset of lytic gene expression (reviewed in references [2,16]). Incorporation of UL14 into the virion tegument promotes the efficient transport of virion-associated VP16 into the nucleus immediately after virus infection [2], and deletion of UL14 significantly alters the targeting of viral capsids to the nuclear pore. The combination of these factors likely contributes to the observed 4-fold delay in the initial expression of the IE genes encoded by ICP0 and ICP4 when cells are infected with UL14-deleted (UL14D) virus [17].

In the present study, we wished to determine whether the HSP-like sequence of UL14 mediated the nuclear

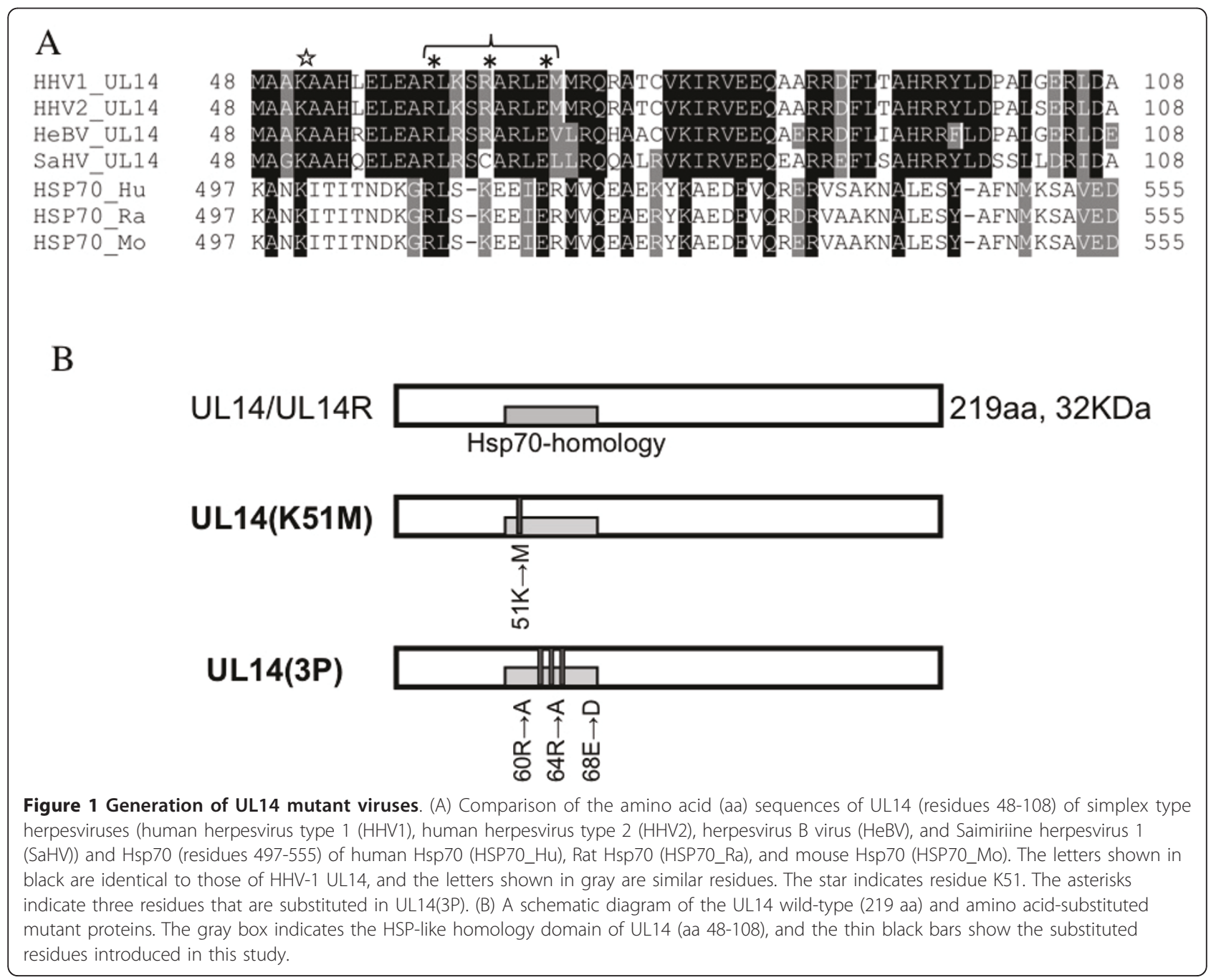


transport of VP16 during the early phase of infection. However, we unexpectedly found that these residues were important for viral replication in the late phase of infection, rather than in the IE phase of infection.

\section{Results}

\section{Construction of UL14 mutant viruses with amino acid} substitutions

To analyze the functions of the UL14 tegument protein, we constructed two mutant viruses with either single or triple amino acid substitutions in UL14. We previously identified a region of homology between the C-terminal domain of mammalian Hsp70 and the $\mathrm{N}$-terminal region of HSV UL14 [9]. The C-terminal region of Hsp70 is thought to modulate substrate recognition and/or maintain its substrate-binding state [18]. Figure 1A shows the sequence alignment between mammalian Hsp70 and herpes virus UL14. K51 is conserved in both $\alpha$-herpes viruses and the sequence ${ }_{60}$ RLKSRARLE $_{68}$ of UL14, which contains a cluster of charged residues, and this region is highly similar to Hsp70 [6,9]. We also incorporated green fluorescence protein (GFP)-conjugated VP16 (VP16GFP) into the genome of these newly generated UL14-mutant viruses (Figure 1B). Successful incorporation of the mutations was verified by sequence analysis. The viruses used in this study were as follows: 1) UL14DVP16GFP (UL14-null mutant); 2) UL14R-VP16GFP (UL14D-rescued virus); 3) UL14(K51M)-VP16GFP (K51 to methionine substitution); 4) UL14(3P)-VP16GFP (R60, R64 to alanine and E68 to aspartic acid substitutions); and 5) UL14(3P)R-VP16GFP (UL14(3P)-rescued virus).

\section{Growth properties of UL14 mutant viruses}

UL14-null virus and mutant viruses all exhibited delayed viral growth in Vero cells. However, the sizes of the plaques differed considerably. We compared the diameters of the plaques formed around the different groups of Vero cells at 72 hours post-infection (h.p.i.) (Figure 2A). UL14R- and UL14(K51M)-VP16GFP formed similar medium-sized plaques, whereas those formed by UL14 (3P)- and UL14D-VP16GFP were smaller. We quantified the diameter of at least 30 plaques for each virus at 36 h.p.i., and there was a significant difference between the plaque sizes of UL14R- and UL14(3P)-VP16GFP viruses $(\mathrm{p}<0.001 ; t$-test) (Figure 2B).

To analyze the impact of these point mutations on viral growth, we investigated both multiple-step and single-step growth kinetics. At a multiplicity of infection (MOI) of 0.001 (PFU/cell), UL14(K51M)-VP16GFP exhibited a $50 \%$ decrease in growth compared to that of the wild-type virus at a viral yield of 100 h.p.i. Moreover, UL14(3P)- and UL14D-VP16GFP viruses revealed further declines of 1-log and 2-logs, respectively (Figure 2C). At an MOI of 3, UL14(K51M)- and UL14(3P)-
VP16GFP exhibited decreased infection of almost 1-log compared to the wild-type viruses, but these decreases were not as large as that exhibited by UL14D-VP16GFP (Figure 2D). These results suggest that both the K51M and the triple mutants affected single- and multiplegrowth kinetics, although the three-point mutation had a more severe impact on viral growth compared to the K51M mutation alone.

\section{Mutation of UL14 altered the intracellular compartmentalization of newly synthesized VP16GFP}

The delayed viral growth exhibited by UL14D- and UL14 (3P)-VP16GFP suggest that mutation of UL14 reduces the efficiency of viral replication and/or propagation in infected cells. We next investigated the intracellular localization of the viral components that comprise viral particles. VP16 is a major tegument protein abundantly expressed in infected cells, and we examined the localization of VP16GFP in HEp-2 cells infected with the different UL14 mutant viruses at an MOI of 3.

Wild-type virus infection led to both cytoplasmic and nuclear localization of VP16GFP (Figure 3A-C). VP16GFP showed a diffuse nuclear pattern followed by the formation of discrete subnuclear foci that grew larger at later times after infection. In the cytoplasm, we observed both diffuse and dotted patterns. Overall, the distribution of VP16 in cells infected with wild-type virus was comparable to that previously reported [19]. Cells infected with UL14(K51M)-VP16GFP or UL14(3P) R-VP16GFP showed a phenotype similar to wild-type virus; however, subnuclear foci were not clearly visualized. There were no substantial differences in the localization of VP16GFP in the mutant virus infected cells compared to wild-type cells (Figure 3G-I, MO).

Cells infected with UL14D-VP16GFP showed a distinct overall morphology and distribution of VP16GFP (Figure 3D-F). At 7 h.p.i, VP16GFP showed diffuse nuclear and cytoplasmic distribution similar to that seen in wild-type-infected cells (Figure 3D). At later time points, VP16GFP had accumulated in the perinuclear region in clumps forming a globular structure (Figure 3E). At 17 h.p.i. VP16GFP was found in the nucleus and the perinuclear region in larger aggregates, and the triple mutant exhibited a phenotype similar to that of UL14D-VP16GFP (Figure 3J-L). Additionally, VP16GFP began to accumulate at the nuclear periphery at later times post-infection.

\section{Intracellular localization of other viral proteins in UL14- mutant viruses}

We next investigated the localization of other viral proteins in cells infected with UL14D-, UL14R-, or UL14 (3P)-VP16GFP. Immunofluorescence analysis revealed that the capsid protein VP5 predominantly localized to 


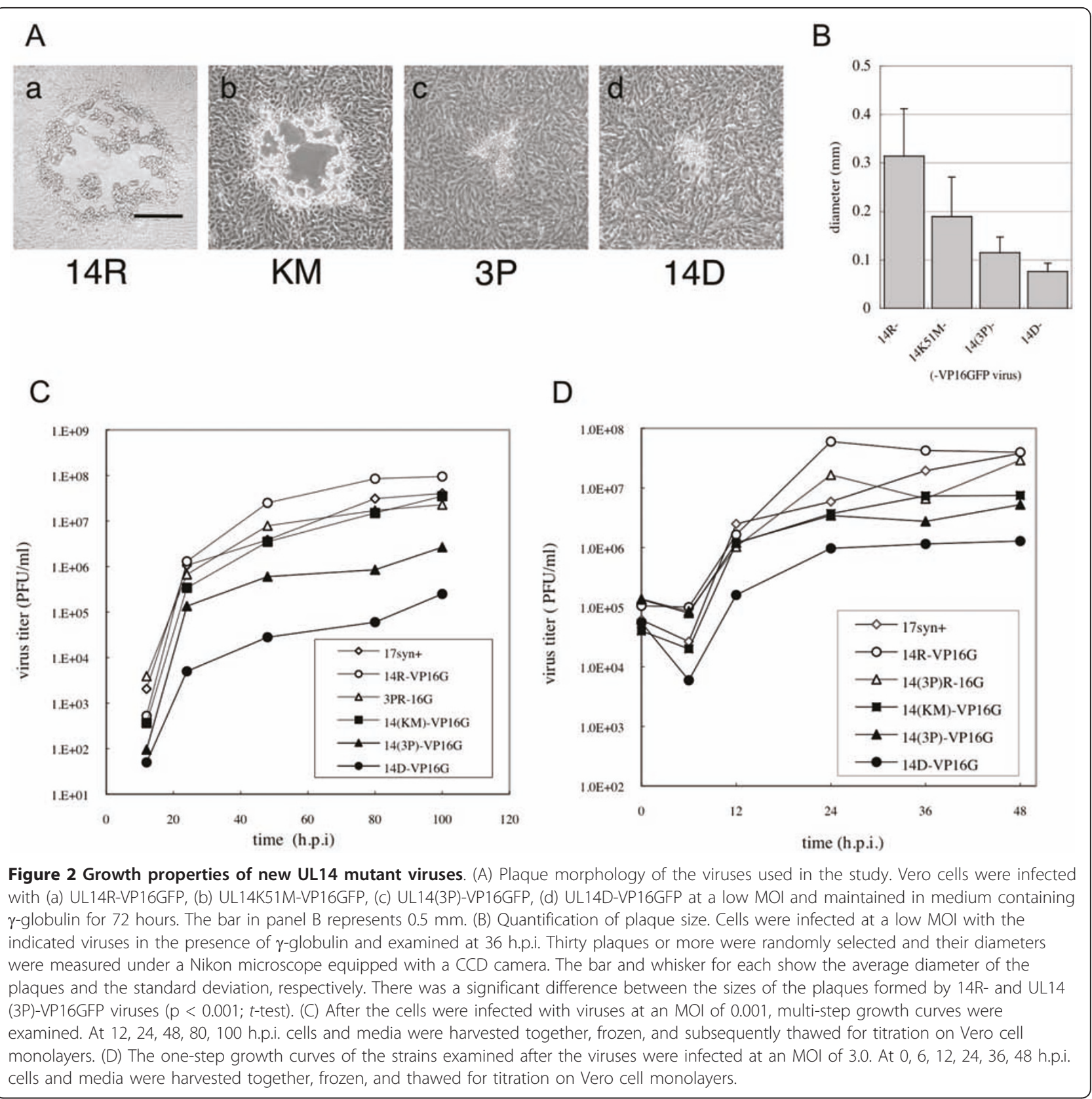

the nucleus from 7 to 17 h.p.i in cells infected with wild-type virus (Figure 4). Moreover, there was little difference in the compartmentalization of VP5 between cells infected with UL14(3P)R- or UL14(3P)-VP16GFP viruses. Both wild-type UL14 and triply mutated UL14 (3P) localized to the nucleus and cytoplasm at 7 h.p.i, and the largest population was seen in the nucleus. Wild-type UL14 in UL14(3P)R-infected cells was distributed throughout the cell at 17 h.p.i., but it was predominantly localized to the nucleus. However, the distinct intracellular localization pattern exhibited by UL14(3P) was also seen in UL14(3P)-16GFP-infected cells at $17 \mathrm{~h}$. p.i. (Figure 4). In both cases, there was the accumulation and aggregation of VP16GFP at the perinuclear region.

We subsequently used immunofluorescence analysis to evaluate the localization of two non-structural viral proteins, ICP8 and UL34. ICP8 is a single-stranded DNAbinding protein, and localizes to sites of viral replication in the cell nucleus [20]. UL34 protein is a tail-anchored type II membrane protein that localizes to the inner nuclear membrane, and it is required for the efficient envelopment of progeny virions at the nuclear envelope $[21,22]$. ICP8 localized to small punctate foci in the nucleus at 7 h.p.i. in UL14R-VP16GFP-infected cells, 


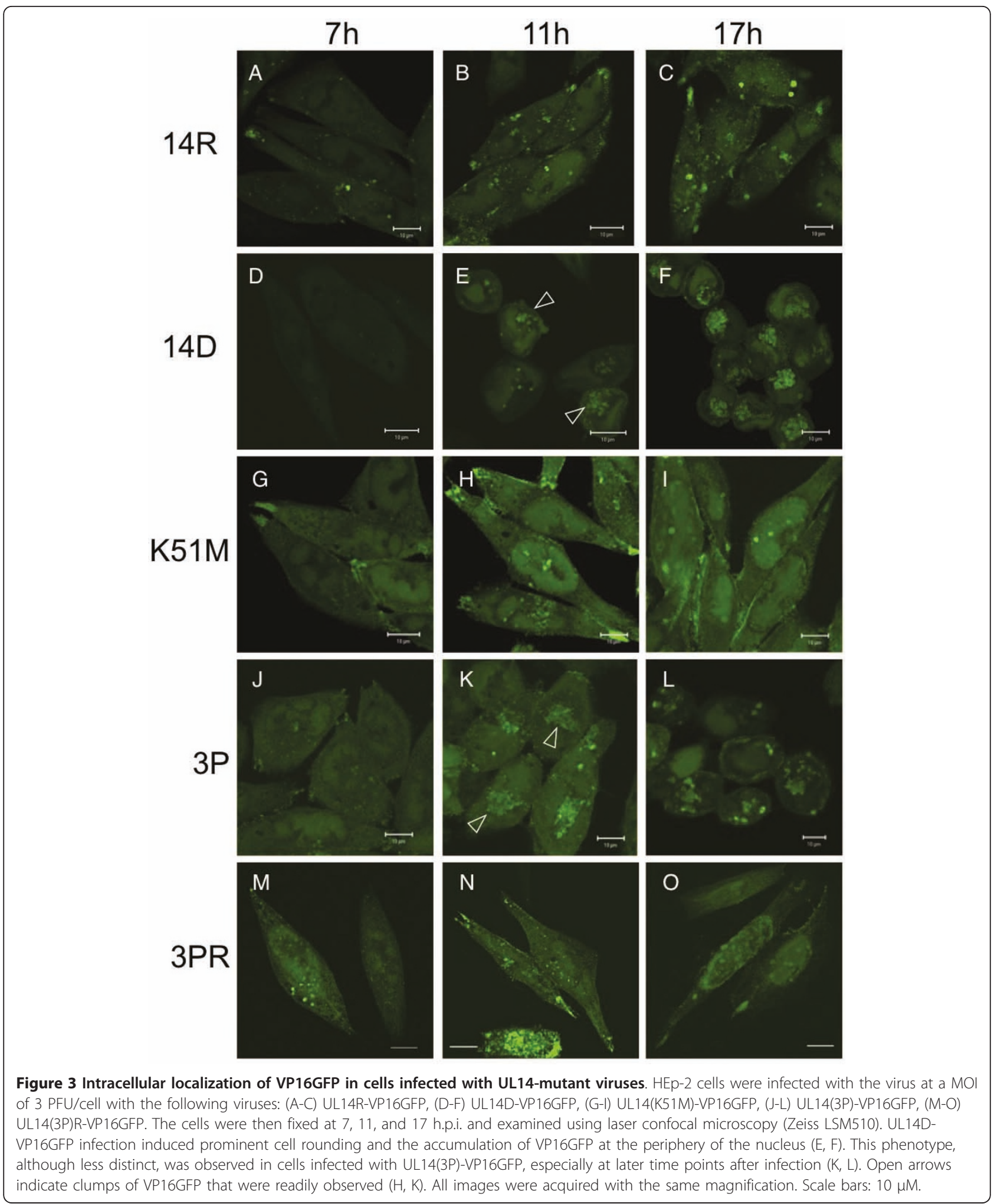

and, by 17 h.p.i. ICP8 localized to a large globular structure (Figure 5). In cells infected with UL14D-VP16GFP, the localization of ICP8 was similar to that seen in UL14R-VP16GFP-infected cells at both time points. In cells infected with either UL14R- or UL14D-VP16GFP,
UL34 localized to the inner nuclear membrane (Figure 5), and we confirmed the localization of UL34 by co-staining with anti-lamin $\mathrm{A} / \mathrm{C}$ (data not shown). Additionally, there was a dramatic morphological change in the nuclei of UL14D-VP16GFP-infected cells, which was accompanied 


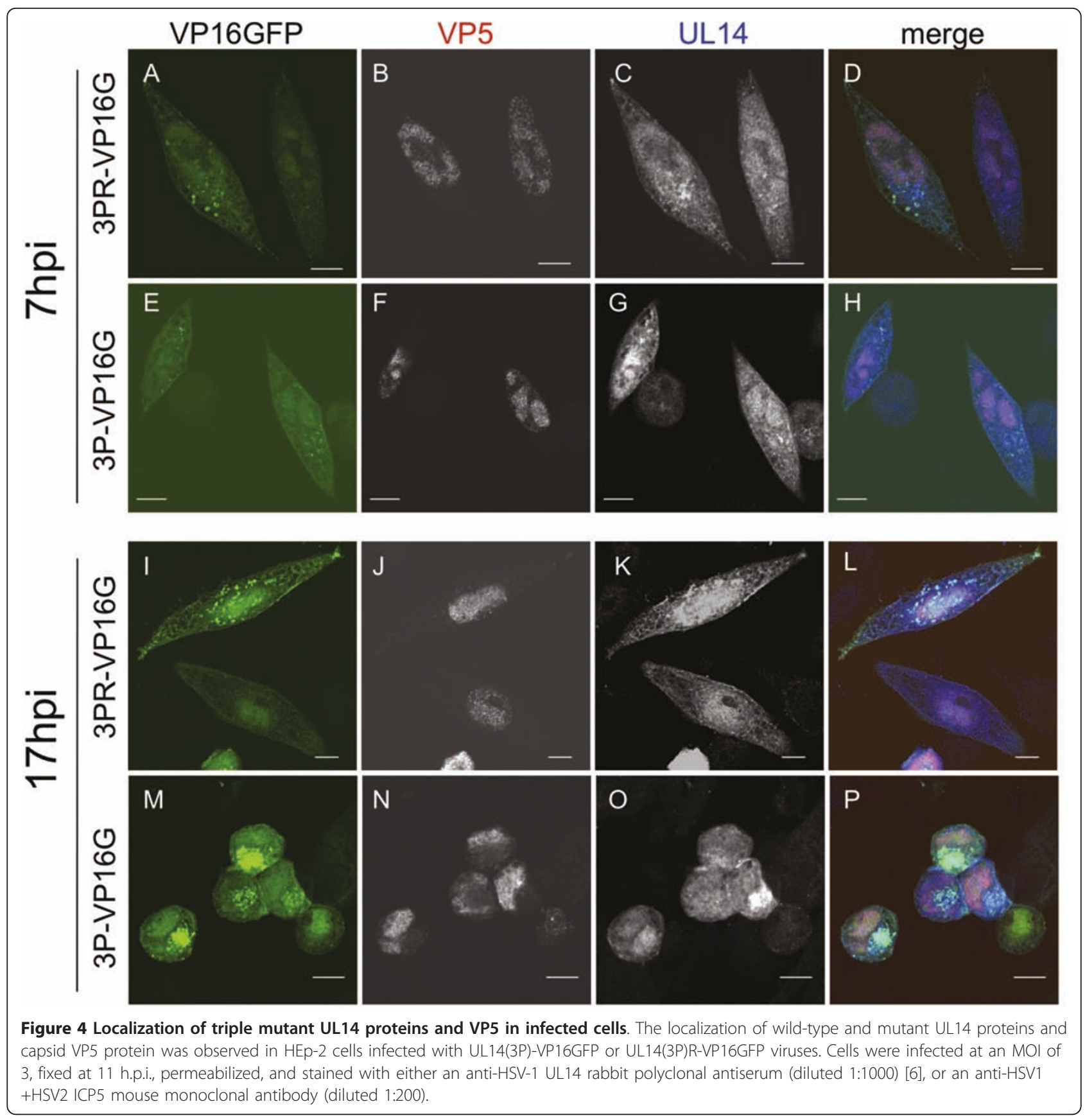

by the formation of the VP16GFP aggregates. These observations suggest that UL14 does not play an important role in the formation of replication compartments in the nucleus. However, the loss of UL14 or expression of UL14 mutants affected the cytoplasmic environment during viral replication.

A UL46 tegument protein (VP11/12) localized to the sites of VP16GFP accumulation

We also investigated the localization of UL46, which strongly associates with VP16 (UL48 protein) and UL47 in the HSV virion [23]. In cells infected with UL14RVP16GFP, a substantial fraction of UL46 localized to cytoplasmic face of the nucleus at 7, 12, and 17 h.p.i., as previously reported (Figure 5 ). The cytoplasmic fraction of UL46 of UL14R-VP16GFP colocalized with VP16GFP around the cell nucleus. Although VP16GFP was also localized to the periphery of the cell, UL46 associated with UL14R-VP16GFP did not localize to that area. In cells infected with UL14D-VP16GFP, the localization of UL46 at 7 h.p.i. was similar to that seen in the UL14Rinfected cells. However, at 12 h.p.i. the dotted 


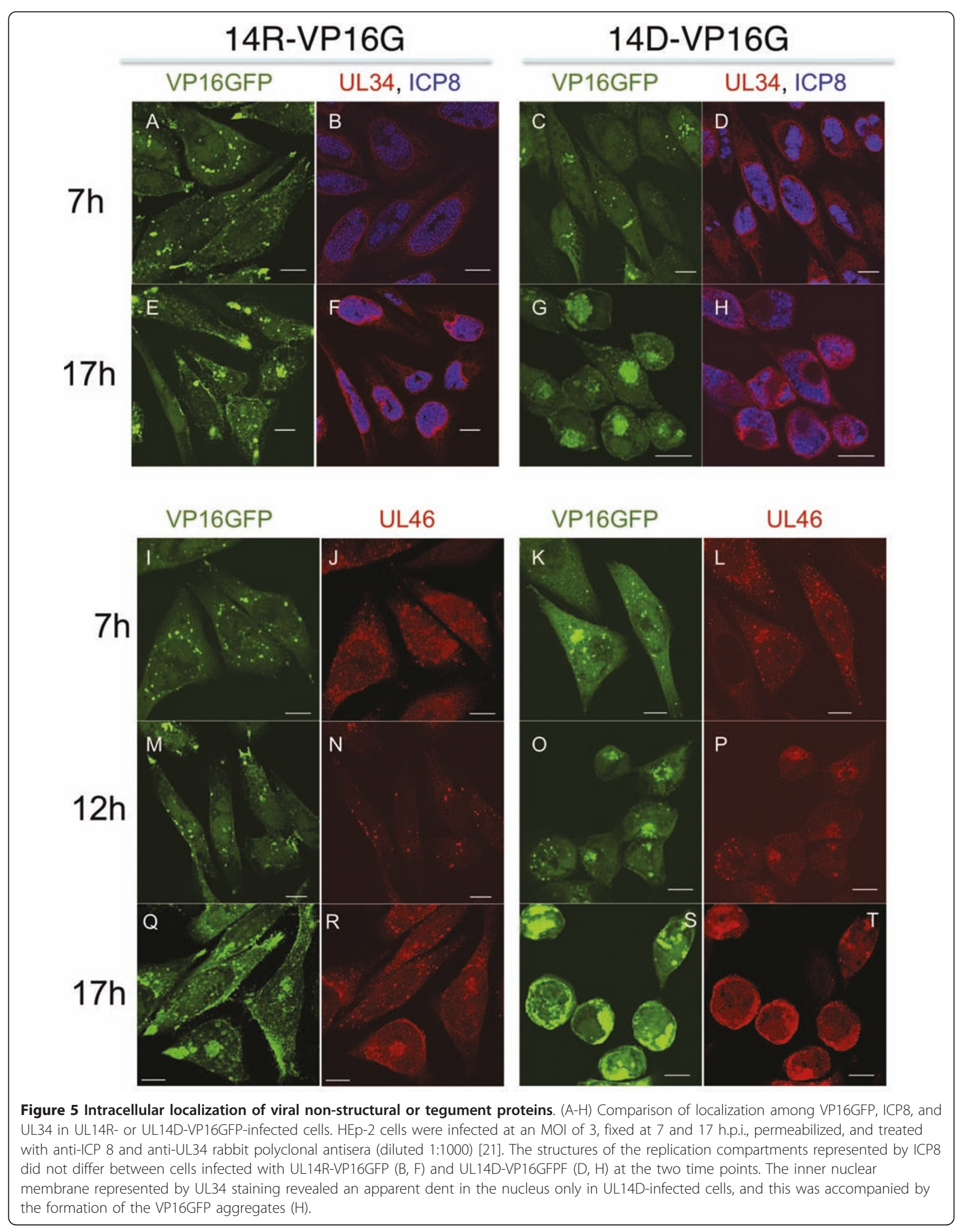


distribution pattern of UL46 in UL14D-VP16GFPinfected cells seen early after infection changed, and UL46 became aggregated at the perinuclear or cytoplasmic space.

\section{Cellular morphological alterations were detected in the UL14-defective virus-infected cells}

There were several morphological changes seen in cells infected with UL14-defective virus at later time points after infection, including spherically shaped cells, shrinkage of the cytoplasmic volume, and an inward curvature of the nucleus (Figure 3E, F, K, L). At these time points, in cells infected with UL14D- or UL14(3P)-VP16GFP virus, the long axes of the cells gradually shortened from 11 h.p.i., but these changes were less apparent at 17 h.p.i. (Figure 3F, L). Therefore, we next investigated if virus infection could alter the distribution of cytoskeletal proteins using immunofluorescence analysis with phalloidin and an anti- $\alpha$-tubulin antibody to detect filamentous actin (F-actin) and microtubules, respectively. In non-infected (mock) cells, there were many actin stress fibers with focal adhesions and a fine mesh of microtubules. Moreover, staining with anti-vinculin demonstrated focal adhesions present at both ends of the actin filaments (data not shown). In UL14R-VP16GFPinfected cells, many stress fibers were detected during infection, and small dots of actin were seen at 17 h.p.i. However, the number of stress fibers and focal adhesions were reduced as viral replication proceeded (Figure 6). Microtubules in UL14R-VP16GFP-infected cells maintained a mesh structure during infection, but unstrained filaments within the mesh were detected at 17 h.p.i. In cells infected with UL14D-VP16GFP, both the disruption of stress fibers and focal adhesions and the formation of cell-to-cell filamentous processes were detected in Factin-stained cells. In addition, an unstrained structure of microtubules was detected at 7 h.p.i., and the microtubules were in a bundled or dotted structure at 17 h.p.i. These observations indicate that infection with virus encoding defective UL14 protein led to the disruption of cytoskeletal organization earlier than similar disruptions in wild type-infected cells.

\section{VP16GFP accumulated at aggresomes in UL14-defective virus-infected cells}

We next investigated whether the sites of VP16GFP and UL46 staining were aggresomes where insoluble or misfolded proteins accumulate. A characteristic morphologic feature of aggresomes is the presence of a vimentin cage surrounding the accumulated proteins at the microtubule-organizing center [24]. Thus, we examined the vimentin structure in HSV-infected cells, and we observed a vimentin cage surrounding the accumulated VP16GFP in UL14D-VP16GFP-infected cells, but the vimentin in the UL14R-VP16GFP-infected cells maintained the cellular cytoskeletal structure (Figure 7). This observation suggests that the aggregated proteins, including VP16GFP and UL46, were isolated from the cell cytoplasm. Our results indirectly demonstrate that the VP16GFP and UL46 present in aggresomes were not associated with the capsid protein VP5 and, consequently, not used for viral replication (Figure 4). Before aggresome formation, misfolded proteins are either associated with molecular chaperones to prevent aggregation and/or facilitate refolding or degraded by the proteasome. However, molecular chaperones and proteasomes often accumulate around the aggresome [25]. Thus, we next examined the localization of the proteasome 20S subunit and the molecular chaperone Hsp70 in UL14R- or UL14D-VP16GFP-infected cells by immunofluorescence. In cells infected with UL14R-VP16GFP, the proteasome $20 S$ subunit predominantly localized to the nucleus and a granular structure was seen in the cytoplasm at 10 h.p.i. (Figure 7). Hsp70 was distributed throughout the nucleus and cytoplasm, and small punctate structures were scattered around the cell. In cells infected with UL14DVP16GFP, the quantitative distributions of both proteins were similar at 10 h.p.i., but the granular structure of the $20 \mathrm{~S}$ subunit was not defined. Additionally, the Hsp70positive structures colocalized with proteasome $20 \mathrm{~S}$ and VP16GFP at the perinuclear region. At 18 h.p.i. the proteasome $20 \mathrm{~S}$ subunit was almost completely localized within the nuclei of the cells infected with UL14R- or UL14D-VP16GFP, and Hsp70 formed ring structures within nuclei (Figure 7), which are defined as virusinduced chaperone-enriched (VICE) domains [26]. VICE domains were detected in both UL14R- and UL14DVP16GFP-infected cells. In addition, some Hsp70 and the proteasome $20 \mathrm{~S}$ subunit colocalized with VP16GFP in the aggresome, but only in the UL14D-VP16GFPinfected cells. The time course of the localization of Hsp70, proteasome, and VP16GFP in the cytoplasm of the UL14D-VP16GFP-infected cells suggests that misfolded and/or functionally impaired VP16GFP were associated with proteasomes and molecular chaperones. However, the increasing mass of accumulated VP16GFP by 18 h.p.i. exceeded the capacity of the cellular protein quality control (PQC) apparatus leading to the formation of aggresomes.

\section{Discussion}

In present study, we showed that mutation of the HSPlike sequence of UL14 altered the intracellular compartmentalization of some HSV-1 tegument proteins, and induced the formation of aggresomes, which included VP16 and UL46, during the late phase of infection. The characteristics of UL14D-VP16GFP infection-including growth delay, early alteration of cell morphology, and 


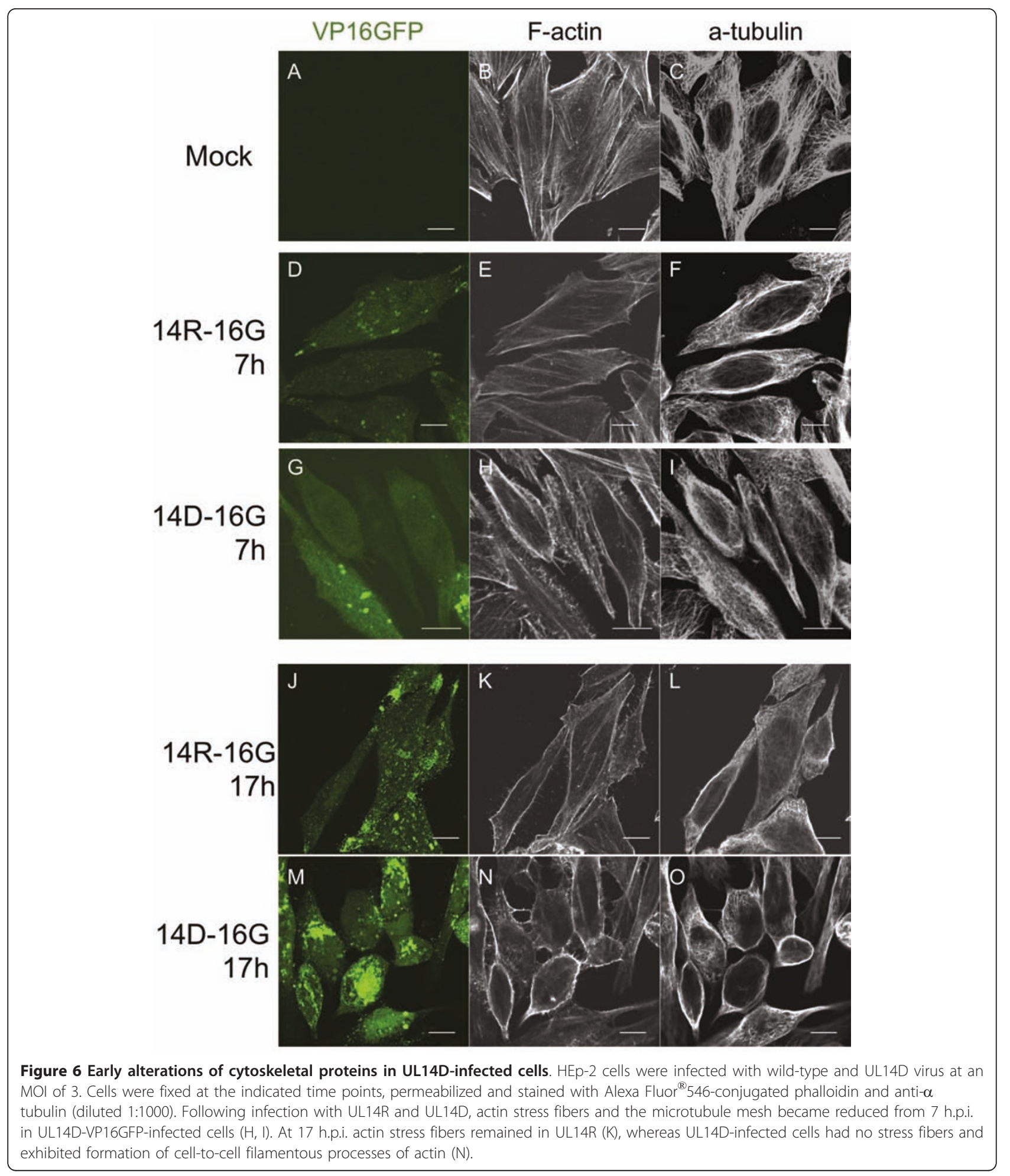

aggresome formation-were clearly observed in cells infected with UL14(3P)-VP16GFP mutant virus, but these phenotypes were not observed in cells infected with UL14(K51M)-VP16GFP virus. These results suggest that a cluster of three charged residues (R60, R64, E68) within the HSP-like sequence of UL14, contribute to viral growth, cell morphology, and compartmentalization of tegument proteins. The ${ }_{60}$ RLKSRARLE $_{68}$ sequence of UL14, which contains a cluster of charged residues, demonstrates sequence similarity to a region of Hsp70 


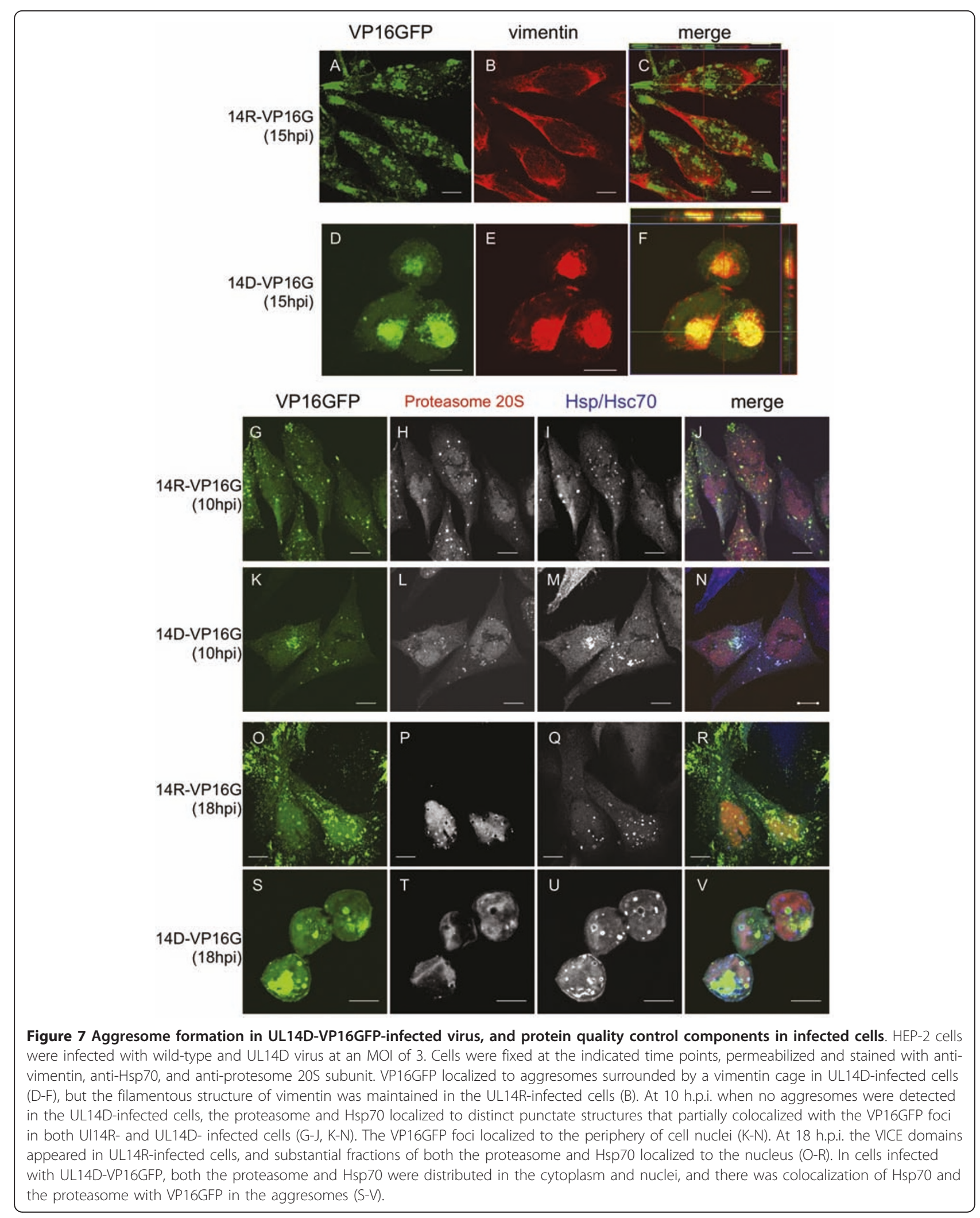


that forms an $\alpha$-helix integrated in the substrate-binding region [8,9]. Mutation of the charged residues in UL14 (3P) may disrupt the interaction of UL14 with other viral proteins, causing a loss of function. However, a direct interaction between UL14 and VP16 has not yet been demonstrated, and further analyses are needed to establish this model.

Immunofluorescence analysis identified several morphological differences between cells infected with UL14D- and UL14R-encoding viruses, and differences were primarily observed in the cytoplasm of cells. The nuclear distribution of the viral replication compartment, VP5 protein, VP16GFP, and VICE domains was unaffected by the deletion or mutation of UL14. Cunningham et al. previously reported that unenveloped capsids accumulated in the cytoplasm of UL14Dinfected cells, and they suggested that UL14 facilitates the addition of the tegument and envelope to assembling virions [6]. Our results also indicate that UL14 is required for events following capsid formation.

The cytoplasmic aggregation of tegument proteins seen in UL14D-infected cells became pronounced as infection progressed. The synthesis of VP16GFP and UL46 proteins increased at approximately the same rate in cells infected with both wild-type and UL14D-encoding virus, as demonstrated by immunoblot analysis (data not shown). However, the growth kinetic analysis indicated that UL14D-VP16GFP-infected cells failed to produce infectious virions at the same rate as UL14RVP16GFP. Moreover, in UL14D-VP16GFP-infected cells, tegument proteins were not efficiently incorporated into the virions. In a previous study, we showed that UL14 of HSV-2 mediated the translocation of both the capsid protein VP26 and the UL33 protein into the nucleus of cells in the absence of infection. UL14 protein also exhibited heat shock protein-like functions when singly expressed. Therefore, we hypothesized that UL14 in infected cells could also play a chaperone-like function and assist the translocation or functional maturation of some tegument proteins.

In this study, we found that HSV-infected cells eventually became rounded and exhibited a loss of adhesion to the extracellular matrix, but these changes became apparent earlier after infection with UL14D- or UL14 (3P)-VP16GFP compared with rescued virus. In particular, we found that some actin stress fibers and focal adhesions of UL14D- and UL14(3P)-VP16GFP-infected cells were decreased at 11 h.p.i. and beyond, but these structures were maintained in mock- and rescued virusinfected cells. No actin polymerization-based protrusions or loss of focal adhesion complexes were seen in normal cells or those infected with rescued virus 11 h.p.i. Therefore, we speculate that UL14 has some role in maintaining the cytoskeletal organization in infected cells.
Aggregates of VP16GFP, UL46, and UL14(3P) were surround by vimentin and sequestered in the cytoplasm, indicating aggresome formation. Aggresomes are formed in cells when there is a large amount of misfolded or insoluble proteins accumulate with cells, but, under normal conditions, aggresome formation is prevented by the activity of the cellular protein quality control (PQC) machinery, which consists of molecular chaperones and proteasomes, which refold or degrade impaired peptides. In the nuclei of HSV-1-infected cells, Weller and colleagues showed that VICE domains contain cellular chaperones, proteasomal components and ubiquitinated proteins, and they suggested that VICE domains function as nuclear PQC centers to remodel or degrade aberrant nuclear proteins interfering with viral infection [27]. Large amounts of newly synthesized viral proteins are present in HSV-infected cells, and some likely require a functional $\mathrm{PQC}$ machinery, and these could be of viral and/or cellular origin. Thus, we hypothesize that the loss of UL14 function increases the amount of misfolded and/or insoluble proteins in the cytoplasm, because some late proteins in particular, such as VP16 and UL46, require the chaperone-like activity of UL14 to mature and/or properly localize within the cell.

\section{Conclusions}

We speculate that the charged residues present in UL14 are important for its chaperone-like functions, and these residues are required for tegument protein maturation and contribute to the sophisticated HSV replication cycle. However, direct interactions between UL14 and VP16 or UL46 have not yet been demonstrated, and the molecular mechanisms underlying the observed cytoskeletal changes remain unclear.

\section{Methods}

Cells

The immortalized African green monkey kidney cell line Vero, the human larynx carcinoma cell line HEp-2, and rabbit skin cells (gifts from B. Roizman) were used. Cells were propagated in Dulbecco's modified Eagle's medium supplemented with $5 \%$ calf serum (Vero cells), $10 \%$ fetal bovine serum (HEp-2 cells), or $5 \%$ fetal bovine serum (rabbit skin cells), each supplemented with 100 $\mathrm{U} / \mathrm{ml}$ penicillin, $100 \mathrm{mg} / \mathrm{ml}$ streptomycin, and $2 \mathrm{mM}$ glutamine. All cells were maintained at $37^{\circ} \mathrm{C}$ with $\mathrm{CO}_{2}$.

\section{Viruses}

HSV-1 wild-type strain 17 syn+, UL14-mutant virus UL14D, and rescued UL14R virus were kindly provided by C. Cunningham. HSV-1 strain MP44 (a syncytiumtype 17syn+ VP16-green fluorescent protein [GFP] virus) was a gift from P. O'Hare. Viral stocks were prepared in Vero or rabbit skin cells, and were subsequently titrated 
separately on Vero cell monolayers. Growth curves were obtained as previously reported [6].

\section{Antibodies}

The following commercial antibodies were used: mouse monoclonal anti-HSV1+HSV2 ICP5 (\#ab6508; Abcam, Inc., Cambridge, MA), mouse monoclonal anti-HSV1 ICP8 (\#ab20194; Abcam), Alexa Fluor ${ }^{\circledR}$ 546-conjugated phalloidin (\#A22283; Invitrogen, Carlsbad, CA), mouse monoclonal anti- $\alpha$-tubulin (\#ab7291; Abcam), mouse monoclonal anti-vimentin clone V9 (Sigma, St. Louis, $\mathrm{MO})$, rabbit polyclonal anti-proteasome $20 \mathrm{~S} \alpha 5$ (\#ab11437; Abcam), mouse monoclonal anti-Hsp70 (\#SPA-810; AssayDesign), Alexa Fluor ${ }^{\circledR} 555$-conjugated goat anti-mouse IgG1, Alexa Fluor ${ }^{\circledR}$ 647-conjugated goat anti-mouse IgG1, Alexa Fluor ${ }^{\circledR} 555$-conjugated goat anti-rabbit IgG, and Alexa Fluor $\left.{ }^{(}\right) 647$-conjugated goat anti-rabbit IgG.

\section{Immunofluorescence}

Cells grown on coverslips were washed with phosphatebuffered saline (PBS) and fixed for $10 \mathrm{~min}$ in $4 \%$ paraformaldehyde in PBS at room temperature. For GFP visualization, the coverslips were mounted directly onto glass slides with PermaFluor (Thermo). For indirect immunofluorescence, fixed cells were permeabilized in $0.1 \%$ Triton X-100 in PBS for $5 \mathrm{~min}$ at room temperature. Each coverslip was inverted onto a droplet $(20 \mathrm{ml})$ of blocking buffer ( $4 \%$ goat serum, $1 \%$ bovine serum albumin in PBS-Tween [0.05\%]) on a clean Parafilm sheet for $30 \mathrm{~min}$ at room temperature. Primary and secondary antibodies (Alexa Fluor; Molecular Probes) were diluted in blocking buffer and reacted for $30 \mathrm{~min}$ at room temperature. To stain with two mouse primary antibodies at once, we used the Zenon ${ }^{\circledR}$ Alexa Fluor ${ }^{\circledR}$ labeling kit (Invitrogen). Samples were examined under a Zeiss LSM510 confocal immunofluorescence microscope.

\section{Viral replication kinetic assay}

Analysis of one-step growth kinetics was performed as described [28] HEp-2 cells were infected with each virus at an MOI of 3 and incubated for 1 hour at $37^{\circ} \mathrm{C}$ to allow for virus adsorption. Any remaining extracellular virus was inactivated by a low-pH treatment ( $\mathrm{pH} 3.0)$, and the cells were incubated at $37^{\circ} \mathrm{C}$ with $5 \% \mathrm{CO}_{2}$. The culture medium was then replaced with newly prepared medium containing $2 \%$ fetal bovine serum. Cells and supernatants were harvested at the indicated times after infection. Virus progeny were titrated on Vero cells by plaque assays.

\section{Abbreviations}

GFP: green fluorescence protein; h.p.i.: hours post-infection; HSP: heat shock protein; HSV: herpes simplex virus; IE: immediate-early; MOI: multiplicity of infection; PBS: phosphate buffered saline; PQC: protein quality control; UL14D: UL14-deleted; UL14(3P): three-point mutated (R60A, R64A, E68D) UL14; VICE: virus induced chaperone enriched.

\section{Acknowledgements}

We thank Joel Baines for the generous gift of the anti-HSV1 UL14 antibody. We would also like to thank Yoko Ushijima, Fumi Goshima, Maki Kamakura, and Chenhong Luo for technical suggestions and discussions, and Tomoko Kunogi and Hiromi Noma for technical assistance. This work was supported by grant-in-aid for scientific research on priority areas (18073007 to YN) and grant-in-aid for Japan Society for the Promotion of Science (JSPS) fellows

(21-6527 to AO) from the Ministry of Education, Culture, Sports, Science and Technology of Japan AO was supported by Research Fellowships for Young Scientists from JSPS.

\section{Author details}

${ }^{1}$ Department of Virology, Graduate School of Medicine, Nagoya University, Tsurumai-cho 65, Showa-ku, Nagoya 466-8550, Japan. ${ }^{2}$ ETH Zurich, Institute of Biochemistry, Schafmattstrasse 18, HPM, ETH Hönggerberg, Zurich, Switzerland.

\section{Authors' contributions}

$A O, Y Y$, and $Y N$ designed the research, $A O, Y Y$, and $Y M$ performed the experimental work, $A O$ conducted the data analysis and drafted the manuscript, and YY, YM, and HK participated in the data analysis and review of the manuscript. All authors read and approved the final manuscript.

\section{Competing interests}

The authors declare that they have no competing interests.

Received: 10 March 2011 Accepted: 26 July 2011

Published: 26 July 2011

\section{References}

1. Dolan A, Jamieson FE, Cunningham C, Barnett BC, McGeoch DJ: The genome sequence of herpes simplex virus type 2. Journal of virology 1998, 72:2010-2021.

2. Roizman B, Knipe DM, Whitley RJ: Herpes Simplex Viruses. In Fields Virology.. Fifth edition. Edited by: Knipe DM, Howley PM, Griffin DE, Lamb RA, Martin MA, Roizman B, Straus SE. Philadelphia, PA: Lippincott Williams 2007:2501-2601.

3. Nishiyama $Y$ : Herpes simplex virus gene products: the accessories reflect her lifestyle well. Rev Med Virol 2004, 14:33-46.

4. McGeoch DJ, Rixon FJ, Davison AJ: Topics in herpesvirus genomics and evolution. Virus Res 2006, 117:90-104.

5. Wada K, Goshima F, Takakuwa H, Yamada H, Daikoku T, Nishiyama Y: Identification and characterization of the UL14 gene product of herpes simplex virus type 2. J Gen Virol 1999, 80(Pt 9):2423-2431.

6. Cunningham C, Davison AJ, MacLean AR, Taus NS, Baines JD: Herpes simplex virus type 1 gene UL14: phenotype of a null mutant and identification of the encoded protein. Journal of Virology 2000, 74:33-41.

7. Yamauchi Y, Wada K, Goshima F, Takakuwa H, Daikoku T, Yamada M, Nishiyama Y: The UL14 protein of herpes simplex virus type 2 translocates the minor capsid protein VP26 and the DNA cleavage and packaging UL33 protein into the nucleus of coexpressing cells. J Gen Virol 2001, 82:321-330

8. Zhu X, Zhao X, Burkholder WF, Gragerov A, Ogata CM, Gottesman ME, Hendrickson WA: Structural analysis of substrate binding by the molecular chaperone DnaK. Science (New York, NY) 1996, 272:1606-1614.

9. Yamauchi Y, Wada K, Goshima F, Daikoku T, Ohtsuka K, Nishiyama Y: Herpes simplex virus type 2 UL14 gene product has heat shock protein (HSP)-like functions. J Cell Sci 2002, 115:2517-2527.

10. Yamauchi Y, Goshima F, Yoshikawa T, Nozawa N, Koshizuka T, Nishiyama Y: Intercellular trafficking of herpes simplex virus type 2 UL14 deletion mutant proteins. Biochemical and Biophysical Research Communications 2002, 298:357-363.

11. Yamauchi Y, Daikoku T, Goshima F, Nishiyama Y: Herpes simplex virus UL14 protein blocks apoptosis. Microbiol Immunol 2003, 47:685-689.

12. Marsden HS, Stow ND, Preston VG, Timbury MC, Wilkie NM: Physical mapping of herpes simplex virus-induced polypeptides. Journal of virology 1978, 28:624-642. 
13. Lemaster S, Roizman B: Herpes simplex virus phosphoproteins. II. Characterization of the virion protein kinase and of the polypeptides phosphorylated in the virion. Journal of virology 1980, 35:798-811.

14. McLean G, Rixon F, Langeland N, Haarr L, Marsden H: Identification and characterization of the virion protein products of herpes simplex virus type 1 gene UL47. The Journal of general virology 1990, 71(Pt 12):2953-2960.

15. Naldinho-Souto R, Browne $H$, Minson T: Herpes simplex virus tegument protein VP16 is a component of primary enveloped virions. Journal of virology 2006, 80:2582-2584.

16. Wysocka J, Herr W: The herpes simplex virus VP16-induced complex: the makings of a regulatory switch. Trends Biochem Sci 2003, 28:294-304.

17. Yamauchi Y, Kiriyama K, Kubota N, Kimura H, Usukura J, Nishiyama Y: The UL14 tegument protein of herpes simplex virus type 1 is required for efficient nuclear transport of the alpha transinducing factor VP16 and viral capsids. Journal of virology 2008, 82:1094-1106.

18. Chou CC, Forouhar F, Yeh YH, Shr HL, Wang C, Hsiao CD: Crystal structure of the C-terminal 10-kDa subdomain of Hsc70. J Biol Chem 2003, 278:30311-30316.

19. La Boissière S, Izeta A, Malcomber S, O'Hare P: Compartmentalization of VP16 in cells infected with recombinant herpes simplex virus expressing VP16-green fluorescent protein fusion proteins. J Virol 2004, 78:8002-8014.

20. Quinlan MP, Chen LB, Knipe DM: The intranuclear location of a herpes simplex virus DNA-binding protein is determined by the status of viral DNA replication. Cell 1984, 36:857-868.

21. Shiba C, Daikoku T, Goshima F, Takakuwa H, Yamauchi Y, Koiwai O, Nishiyama Y: The UL34 gene product of herpes simplex virus type 2 is a tail-anchored type II membrane protein that is significant for virus envelopment. J Gen Virol 2000, 81:2397-2405.

22. Reynolds AE, Ryckman BJ, Baines JD, Zhou Y, Liang L, Roller RJ: U(L)31 and $\mathrm{U}(\mathrm{L}) 34$ proteins of herpes simplex virus type 1 form a complex that accumulates at the nuclear rim and is required for envelopment of nucleocapsids. Journal of Virology 2001, 75:8803-8817.

23. Kato K, Daikoku T, Goshima F, Kume H, Yamaki K, Nishiyama Y: Synthesis, subcellular localization and VP16 interaction of the herpes simplex virus type 2 UL46 gene product. Arch Virol 2000, 145:2149-2162

24. Tyedmers J, Mogk A, Bukau B: Cellular strategies for controlling protein aggregation. Nat Rev Mol Cell Biol 2010, 11:777-788.

25. Garcia-Mata R, Bebok Z, Sorscher EJ, Sztul ES: Characterization and dynamics of aggresome formation by a cytosolic GFP-chimera. J Cell Biol 1999, 146:1239-1254.

26. Burch AD, Weller SK: Nuclear sequestration of cellular chaperone and proteasomal machinery during herpes simplex virus type 1 infection. J Virol 2004, 78:7175-7185.

27. Livingston CM, Ifrim MF, Cowan AE, Weller SK: Virus-Induced ChaperoneEnriched (VICE) domains function as nuclear protein quality control centers during HSV-1 infection. PLOS Pathog 2009, 5:e1000619.

28. Nozawa N, Kawaguchi Y, Tanaka M, Kato A, Kato A, Kimura H, Nishiyama Y: Herpes simplex virus type 1 UL51 protein is involved in maturation and egress of virus particles. Journal of Virology 2005, 79:6947-6956.

doi:10.1186/1743-422X-8-365

Cite this article as: Ohta et al.: Herpes simplex virus type 1 UL14

tegument protein regulates intracellular compartmentalization of major tegument protein VP16. Virology Journal 2011 8:365.

\section{Submit your next manuscript to BioMed Central and take full advantage of:}

- Convenient online submission

- Thorough peer review

- No space constraints or color figure charges

- Immediate publication on acceptance

- Inclusion in PubMed, CAS, Scopus and Google Scholar

- Research which is freely available for redistribution

Submit your manuscript at www.biomedcentral.com/submit
Biomed Central 